\title{
Estratégias para o monitoramento da saúde do trabalhador de enfermagem
}

\author{
Strategies for monitoring the nursing worker's health \\ Estrategias de seguimiento de la salud del trabajador de enfermería
}

\author{
Márcia Lima da Cunha \\ ORCID: https://orcid.org/0000-0002-5489-7082 \\ Universidade do Estado do Rio de Janeiro, Brasil \\ E-mail: marcia.cunha@ rededor.com.br \\ Larissia Admá de Souza Pereira \\ ORCID: https://orcid.org/0000-0002-4242-2354 \\ Universidade do Estado do Rio de Janeiro, Brasil \\ E-mail: laripereira2006@yahoo.com.br \\ Raquel Silva Gomes \\ ORCID: https://orcid.org/0000-0002-2131-2009 \\ Universidade do Estado do Rio de Janeiro, Brasil \\ E-mail: raquelijgomes@gmail.com \\ Raquel Veiga Baptista \\ ORCID: https://orcid.org/0000-0002-5150-8299 \\ Universidade do Estado do Rio de Janeiro, Brasil \\ E-mail: raquel.veiga.baptista@hotmail.com \\ Bruna de Jesus Freitas \\ ORCID: https://orcid.org/0000-0001-9279-2946 \\ Universidade do Estado do Rio de Janeiro, Brasil \\ E-mail: brunafreitas1997@gmail.com \\ Magda Guimarães de Araújo Faria \\ ORCID: https://orcid.org/0000-0001-9928-6392 \\ Universidade do Estado do Rio de Janeiro, Brasil \\ E-mail: magda.faria@live.com \\ Patrícia Campos Pavan Baptista \\ ORCID: https://orcid.org/0000-0003-1433-6456 \\ Universidade do Estado do Rio de Janeiro, Brasil \\ E-mail: pavanpati@usp.br \\ Cristiane Helena Gallasch \\ ORCID: https://orcid.org/0000-0002-0823-0818 \\ Universidade do Estado do Rio de Janeiro, Brasil \\ E-mail: cristiane.gallasch@gmail.com
}

\begin{abstract}
Resumo
Objetivo: analisar as publicações nacionais e internacionais sobre estratégias para monitoramento da saúde do trabalhador de enfermagem. Método: revisão integrativa da literatura. As buscas foram realizadas nas bases Literatura Latino-Americana e do Caribe em Ciências da Saúde, Medical Literature Analysis and Retrieval System Online, Cumulative Index to Nursing and Allied Health Literature e Scopus, usando descritores. A busca resultou em 687 estudos. Após análise dos critérios de inclusão e exclusão, foram selecionados 06 estudos. Resultados: foram encontradas seis publicações que atenderam aos critérios do estudo, que indicaram a existência apenas da ferramenta sistema de monitoramento da saúde dos trabalhadores de enfermagem. Conclusão: é evidente à escassez de trabalhos sobre a referida temática, foi identificada a necessidade de implementar ferramentas tecnológicas para favorecer estratégias preventivas para o monitoramento das notificações de doenças dos trabalhadores de enfermagem.

Palavras-chave: Saúde em saúde; Profissionais de enfermagem do trabalhador; Enfermagem; Processo saúde-doença; Vigilância.

Abstract

Objective: to analyze national and international publications on strategies for monitoring the health of nursing workers. Method: integrative literature review. The searches were carried out on the bases of Latin American and Caribbean Literature in Health Sciences, Medical Literature Analysis and Retrieval System Online, Cumulative Index to Nursing and Allied Health Literature and Scopus, using descriptors. The search resulted in 687 studies. After analyzing the inclusion and exclusion criteria, 06 studies were selected. Results: six publications were found that met the study criteria, which indicated the existence only of the nursing workers' health monitoring system tool. Conclusion: it is evident from the scarcity of studies on this subject, the need to implement technological tools to favor preventive strategies for monitoring the notifications of illnesses of nursing workers was identified.
\end{abstract}


Keywords: Health in health; Nursing professionals of the worker; Nursing; Health-disease process; Surveillance.

\section{Resumen}

Objetivo: analizar publicaciones nacionales e internacionales sobre estrategias de vigilancia de la salud del personal de enfermería. Método: revisión integradora de la literatura. Las búsquedas se realizaron sobre las bases de Literatura Latinoamericana y Caribeña en Ciencias de la Salud, Sistema de Análisis y Recuperación de Literatura Médica en Línea, Índice Acumulativo de Literatura de Enfermería y Afines en Salud y Scopus, utilizando descriptores. La búsqueda resultó en 687 estudios. Después de analizar los criterios de inclusión y exclusión, se seleccionaron 06 estudios. Resultados: se encontraron seis publicaciones que cumplieron con los criterios del estudio, que indicaron la existencia únicamente de la herramienta del sistema de vigilancia de la salud del trabajador de enfermería. Conclusión: se evidencia la escasez de estudios sobre este tema, identificando la necesidad de la implementación de herramientas tecnológicas que favorezcan las estrategias preventivas para el seguimiento de los informes de enfermedad entre los trabajadores de enfermería.

Palabras clave: Salud en salud; Enfermería del trabajador; Enfermería; Proceso salud-enfermedad; Vigilancia.

\section{Introduçãa}

A saúde, como direito universal e dever do Estado, é uma conquista da população brasileira expressa na Constituição Federal de 1988 e regulamentada pela Lei Orgânica da Saúde (Brasil, 1988). Nessa perspectiva, destaca-se que a saúde de uma população está diretamente ligada a seu local de trabalho, no cenário de uma sociedade que passa a maior parte de seu tempo dentro de instituições corporativas.

Historicamente, a saúde do trabalhador configura-se como um campo de conhecimentos interdisciplinares, técnicos, sociais, políticos e humanos, voltados para intervir nas relações de trabalho que provocam doenças e agravos à saúde dos indivíduos, com marco referencial brasileiro na Saúde Coletiva, ou seja, com foco na promoção, prevenção e vigilância em saúde influenciada pela Medicina social latino-americana e pelo Movimento Operário Italiano (Gomes, et al., 2018).

Desde a década de 1990, a Vigilância em Saúde do Trabalhador no Brasil integra o Sistema Único de Saúde (SUS), com ações que desenvolvidas nos âmbitos urbano e rural, por Secretarias de Saúde dos Estados, do Distrito Federal e dos Municípios (Brasil, 1988), sendo reiterada na proposta da Política Nacional de Saúde do Trabalhador e Trabalhadora (PNSTT) (Brasil, 2012). Além disso, o monitoramento da saúde do trabalhador foi estabelecido pela da Norma Regulamentadora número 7 (NR-7), por meio da obrigatoriedade da elaboração e execução do Programa de Controle Médico de Saúde Ocupacional PCMSO (Brasil, 1994).

Tratando-se de exposição, os trabalhadores da área da saúde estão expostos, em geral, a inúmeros agentes causadores de doenças, tendo em vista o seu objeto de trabalho. Tal realidade torna indispensável o conhecimento de medidas de proteção individuais e coletivas, além do desenvolvimento de ações de promoção e vigilância em saúde nesta população, caracterizadas por prevenção, rastreamento e diagnóstico da situação de saúde do trabalhador (Trindade, et al., 2017).

Nesse aspecto, os trabalhadores de enfermagem estão entre as categorias mais acometidas por doenças ocupacionais na área da saúde, em geral caracterizadas por afecções osteomusculares, cardiovasculares, respiratórias, transtornos emocionais e comportamentais, além da exposição a fluidos biológicos, doenças infectocontagiosas e parasitárias, entre outras7. Ademais, o absenteísmo nessa categoria é extremamente representativo, sendo mais elevado quando comparado a índices de trabalhadores em geral (Santana, et al., 2017).

Portanto, há necessidade de conhecer, realizar e manter ações de vigilância em saúde do trabalhador de enfermagem, uma vez que estão constantemente expostos a diversos determinantes e condicionantes em saúde que os expõem a riscos frequentes. É necessário um trabalho de monitoramento de dados que influencie na diminuição de ocorrências de agravos e na melhora na qualidade de vida desses profissionais, que embora promovam o cuidado em sua atuação profissional, nas suas vidas, em geral, não se cuidam ou não possuem ambientes de trabalho que permitam esse autocuidado (Guimarães \& Felli, 2016). 
Entende-se, com isso, que é relevante buscar como acontece, na ótica científica e contemporânea, o monitoramento da saúde do trabalhador de enfermagem no Brasil. Neste sentido, questiona-se: quais as estratégias disponíveis para acompanhamento do processo de adoecimento do trabalhador de enfermagem?

Assim, a presente investigação com o objetivo avaliar as informações disponíveis na literatura sobre as estratégias para monitoramento da saúde do trabalhador de enfermagem.

\section{Metodologia}

Trata-se de uma revisão integrativa de literatura, descrita como método de pesquisa que permite a síntese e aprofundamento de pesquisas já existentes e relevantes sobre um determinado tema, as quais corroboram para melhor descrição sistematizada sobre o fenômeno estudado (Mendes, et al., 2020).

O estudo foi composto pelas seguintes etapas:(1) identificação do tema e definição da questão norteadora, (2) delineamento dos bancos de dados da pesquisa científica e definição dos critérios de inclusão e exclusão, (3) definição das informações a serem extraídas e categorização dos estudos selecionados, (4) avaliação dos estudos incluídos, (5) interpretação dos resultados e apresentação da síntese do conhecimento (Ercoli, et al., 2020).

A partir do questionamento: "quais as estratégias disponíveis para acompanhamento do processo de adoecimento do trabalhador de enfermagem?", foram definidos descritores para busca em nos idiomas português e inglês, conforme padronizado pela Biblioteca Virtual em Saúde (BVS), utilizando os Descritores em Ciências da Saúde (DeCS) e seus equivalentes definidos pelo Medical Subject Headings (MeSH): "Processo Saúde-Doença", "Vigilância em Saúde", "Vigilância em Saúde Pública" e "Profissionais de Enfermagem", "Enfermagem", "Saúde do Trabalhador", "Enfermagem do Trabalho". Além desses, foram utilizadas também as palavras-chave "Adoecer", "Adoecimento", "Vigilância da Saúde," "Monitoramento em saúde", "Acompanhamento em saúde".

A seguir, entre outubro e novembro de 2020, desenvolveu-se a busca nas seguintes bases Literatura Latino-Americana e do Caribe em Ciências da Saúde (LILACS), PubMed, Cumulative Index to Nursing and Allied Health Literature (CINAHL) e Scopus. A fim de restringir a busca ao objeto de estudo, evitando a captura de informações irrelevantes ao estudo (Moher, et al., 2009). Foram utilizados os operadores boleanos [AND] e [OR], como apresentado na Figura 1. Não foi estabelecido um recorte temporal ou idioma de publicação, considerando a contemporaneidade da temática e buscando abarcar o maior número de produções possíveis.

Foram incluídos estudos originais, envolvendo o monitoramento da saúde do trabalhador de enfermagem, publicados na íntegra, uma vez que se considerou necessária a avaliação detalhada da estratégia descrita. Foram excluídos estudos teóricos ou de revisão, em duplicidade, não disponíveis na íntegra ou que envolvessem a temática da vigilância à saúde de trabalhadores de outros campos profissionais.

Os resultados da busca e seleção dos manuscritos foram submetidos à dupla checagem de busca, seleção e leitura, e organizados de acordo com as recomendações do Preferred Reporting Items for Systematic Reviews and Meta-Analyses (PRISMA)12, como demonstrado na Figura 1. 
Figura 1. Preferred Reporting Items for Systematic Reviews and Meta-Analyses.

\begin{tabular}{|c|c|c|}
\hline 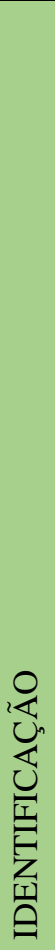 & \multicolumn{2}{|c|}{ 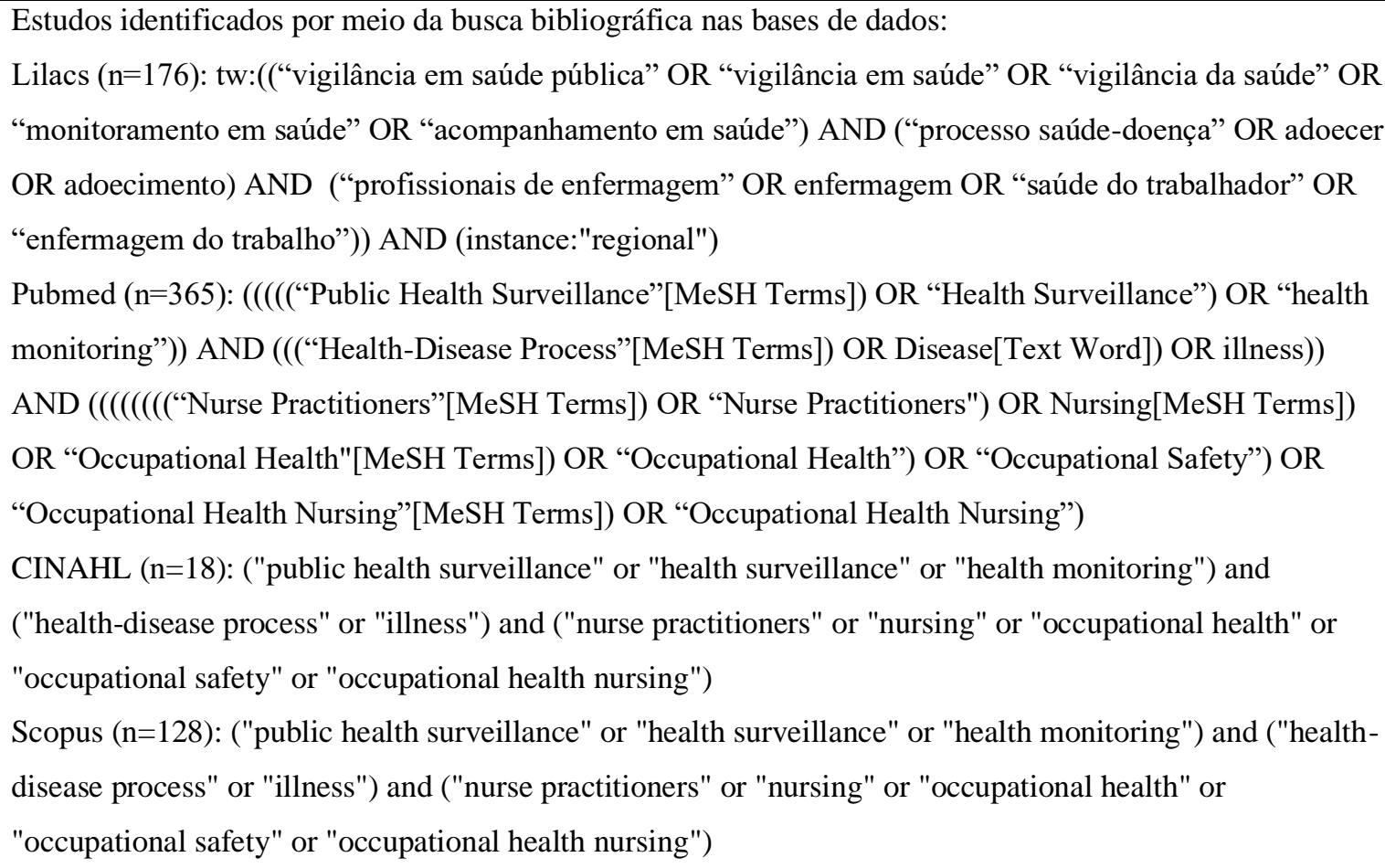 } \\
\hline & $\begin{array}{l}\text { Estudos rastreados: } \\
\text { Estudos originais, literatura branca, } \\
\text { envolvendo seres humanos: Lilacs }(\mathrm{n}=176) ; \\
\text { Pubmed }(\mathrm{n}=365) \text {; Cinahl }(\mathrm{n}=18) ; \text { Scopus } \\
(\mathrm{n}=128) \\
\mathrm{n}=687\end{array}$ & $\begin{array}{l}1^{\text {a }} \text { exclusão: Duplicidade; adequação segundo a } \\
\text { leitura dos títulos. } \\
\text { Critérios: estudos que não se relacionam à temática. } \\
\text { Lilacs ( } \mathrm{n}=153) \text {; Pubmed ( } \mathrm{n}=364) \text {; Cinahl }(\mathrm{n}=18) \\
\text { Scopus ( } \mathrm{n}=128) \\
\text { n: } 663\end{array}$ \\
\hline 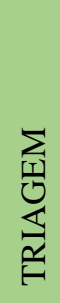 & $\begin{array}{l}\text { Resumos avaliados: } \\
\text { Trabalhos na área da saúde, artigos } \\
\text { originais, artigos que contemplam o objeto } \\
\text { de estudo. } \\
\text { n=24 }\end{array}$ & $\begin{array}{l}2^{a} \text { exclusão: Estudos excluídos a partir da leitura dos } \\
\text { resumos } \\
\text { Critérios: } \\
\text { Estudos que não originais: } \\
\text { Lilacs ( } \mathrm{n}=1) \text {; Pubmed }(\mathrm{n}=2)\end{array}$ \\
\hline 空 & $\begin{array}{l}\text { Estudos em texto completo avaliados para } \\
\text { elegibilidade: verificação de critérios de } \\
\text { inclusão }\end{array}$ & $\begin{array}{l}3^{\text {a }} \text { Exclusão: realizada a partir da leitura do texto } \\
\text { completo } \\
\text { Critérios: não envolvem a população alvo; não } \\
\text { contribuem para o objeto de estudo. } \\
\mathrm{n}=15\end{array}$ \\
\hline 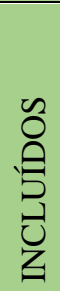 & $\begin{array}{l}\text { Amostra final: Estudos incluídos na síntese } \\
\text { que apresentam evidências psicométricas } \\
\text { sobre instrumentos de medida em saúde do } \\
\text { trabalhador } \\
n=6\end{array}$ & \\
\hline
\end{tabular}


Após procedimento de busca e aplicação dos critérios de inclusão e exclusão, utilizou-se o software Microsoft Office Excel 2010 para viabilizar o agrupamento das informações, utilizando-se as variáveis: autor, ano de publicação, país de realização do estudo, objetivos, método empregado, principais resultados obtidos.

\section{Resultados e Discussão}

A amostra de estudos foi composta de seis publicações, que demonstraram que o tema e a população pesquisados passaram a ser descritos pela literatura a partir da década de 2000, com todos os estudos desenvolvidos no Brasil13-18. Os dados dos manuscritos selecionados são apresentados no Quadro 1.

Quadro 1: Estudos incluídos na revisão integrativa de acordo com país, ano, delineamento e resultados. Rio de Janeiro, RJ, Brasil, 2020. (N=6)

\begin{tabular}{|c|c|c|c|}
\hline País / Ano & Objetivo & Delineamento & Resultados \\
\hline Brasil, 2008 & $\begin{array}{c}\text { Identificar as causas que motivaram o } \\
\text { afastamento do trabalho de profissionais } \\
\text { de enfermagem de um hospital } \\
\text { universitário do oeste do Paraná. }\end{array}$ & QT, DE, EX & $\begin{array}{l}\text { Descreve causas de afastamento dos profissionais de } \\
\text { enfermagem, mais incidentes em Unidades de Terapia } \\
\text { Intensiva (UTI geral e neonatal). Discute a possível } \\
\text { associação entre a rotina estressante do ambiente } \\
\text { hospitalar, bem como as condições de trabalho às quais } \\
\text { esses trabalhadores estão expostos, contribuindo para o } \\
\text { adoecimento. }\end{array}$ \\
\hline Canadá, 2008 & $\begin{array}{c}\text { Fundir os dados administrativos de } \\
\text { Saúde Ocupacional }(\mathrm{OH}) \text { e Recursos } \\
\text { Humanos }(\mathrm{RH}) \text { para descrever os } \\
\text { motivos de absenteísmo entre os } \\
\text { funcionários do hospital e considerar as } \\
\text { vantagens e desvantagens de usar esses } \\
\text { dados combinados para a vigilância dos } \\
\text { profissionais de saúde. }\end{array}$ & Qualitativo & $\begin{array}{c}\text { Esta pesquisa destaca a questão do absenteísmo entre os } \\
\text { profissionais de saúde e a necessidade de melhorar a } \\
\text { notificação de doenças e lesões para OH para a eficácia da } \\
\text { vigilância. Um sistema de vigilância de saúde pública que } \\
\text { monitora as visitas da saúde do trabalhador entre os } \\
\text { profissionais de saúde pode facilitar a prática de saúde } \\
\text { pública. }\end{array}$ \\
\hline Brasil, 2011 & $\begin{array}{l}\text { Conhecer a carga de trabalho e seus } \\
\text { fatores determinantes, buscando } \\
\text { identificar possíveis relações entre as } \\
\text { condições de trabalho e o possível } \\
\text { impacto para a saúde dos trabalhadores. }\end{array}$ & QL, DE, EX & $\begin{array}{l}\text { Os resultados indicam a presença de carga física, carga } \\
\text { cognitiva e carga psíquica; processo de comunicação } \\
\text { inadequado; equipamentos obsoletos; número excessivo } \\
\text { de usuários e estrutura frágil para lidar com absenteísmo. }\end{array}$ \\
\hline Brasil, 2013 & $\begin{array}{c}\text { Analisar as cargas de trabalho, processos } \\
\text { de desgaste e absenteísmo por doença } \\
\text { entre trabalhadores de enfermagem de } \\
\text { um hospital universitário da região } \\
\text { Centro-Oeste. }\end{array}$ & QT, DE, EX & $\begin{array}{l}\text { As cargas fisiológicas e psíquicas foram as mais } \\
\text { representativas, com } 37 \text { e } 36 \% \text {, respectivamente. Os } \\
\text { achados deste estudo permitem evidenciar o impacto das } \\
\text { doenças ocupacionais no absenteísmo dos trabalhadores } \\
\text { de enfermagem, podendo ser utilizado para demonstrar a } \\
\text { importância dos investimentos institucionais na vigilância } \\
\text { da saúde dos trabalhadores. }\end{array}$ \\
\hline Brasil, 2013 & $\begin{array}{l}\text { Caracterizar as cargas e os desgastes } \\
\text { vivenciados em um hospital } \\
\text { universitário de Curitiba. }\end{array}$ & QT, DE, EX & $\begin{array}{l}\text { Os resultados, captados a partir do estudo prévio } \\
\text { intitulado "Sistema de monitoramento da saúde do } \\
\text { trabalhador de enfermagem" (SIMOSTE), demonstram } \\
\text { que o gênero feminino foi o mais acometido (85,9\%); os } \\
\text { profissionais mais afetados foram os auxiliares de } \\
\text { enfermagem (53,1\%). O maior número de afastamentos } \\
\text { ocorreu por doenças do sistema osteoarticular (25,2\%). } \\
\text { Entre as cargas apresentadas, as mais expressivas foram as } \\
\text { mecânicas e fisiológicas, com 33,06\%, cada. Podem } \\
\text { subsidiar estratégias de intervenção nas políticas } \\
\text { direcionadas à saúde do trabalhador, para assegurar uma } \\
\text { melhor qualidade de vida a este profissional e, por } \\
\text { consequência, promover melhorias na qualidade da } \\
\text { assistência prestada ao usuário. }\end{array}$ \\
\hline
\end{tabular}




\begin{tabular}{|c|c|c|c|}
\hline Brasil, 2014 & $\begin{array}{c}\text { Identificar os agravos ocorridos em } \\
\text { trabalhadores de enfermagem por meio } \\
\text { do Sistema de Monitoramento da Saúde } \\
\text { dos Trabalhadores de Enfermagem } \\
\text { (SIMOSTE) e descrever suas } \\
\text { consequências. }\end{array}$ & QT, DE, EX & $\begin{array}{c}\text { Identificadas } 1.847 \text { ocorrências em seis meses, } \\
\text { especialmente por licenças médicas, acidentes de trabalho } \\
\text { com e sem afastamentos, atendimentos psiquiátricos e } \\
\text { psicoterápicos. Indica-se a necessidade de } \\
\text { desenvolvimento de novas ações de vigilância em saúde } \\
\text { voltadas para a notificação dos acidentes e das doenças } \\
\text { relacionadas ao trabalho, além da prevenção dos agravos. }\end{array}$ \\
\hline
\end{tabular}

Legenda: QT: quantitativo; QL: qualitativo; DE: descritivo; EX: exploratório. Fonte: Autoras (2020).

São apresentados estudos realizados no Sul, no Sudeste e no Centro-oeste do país, com predomínio de identificação de cargas de trabalho fisiológicas e as psíquicas, além de maior incidência de afastamentos em unidades de cuidado intensivo. Apenas uma ferramenta é citada como possível estratégia para sistematização do monitoramento da saúde do trabalhador de enfermagem - o Sistema de Monitoramento da Saúde do Trabalhador de Enfermagem (SIMOSTE®) (Santana, et al., 2013; Ferigollo, et al., 2016).

Foram reconhecidos dados e discussões acerca da vigilância no trabalho do profissional de saúde, além do destaque da importância ao seu monitoramento. Porém, apesar de dados de Vigilância em Saúde do Trabalhador (Visat) serem essenciais para uma intervenção eficaz, não são observados dados longitudinais, o que realmente caracterizaria o monitoramento dos dados e que poderiam influenciar diretamente ações de promoção à saúde e prevenção de agravos relacionados ao trabalho.

O monitoramento da saúde do trabalhador de enfermagem é essencial para o desenvolvimento de indicadores, que possibilitem a determinação dos riscos do ambiente de trabalho, assim como as cargas e processos de desgaste, a fim de delinear o perfil epidemiológicos dos trabalhadores e as condições daquele ambiente laboral.

Apesar dos estudos descritos terem sido realizados no Brasil, a problemática de desgaste do profissional de enfermagem, especialmente por distúrbios osteomusculares e transtornos mentais é descrita mundialmente, com exposição a cargas biológicas, fisiológicas e psíquicas. (Silva, 2011; Bernardes, et al., 2014).

Além disso, equipamentos obsoletos, dimensionamento de pessoal insuficiente e dificuldades para lidar com o absenteísmo também são relatados como elementos que favorecem o adoecimento de trabalhadores de enfermagem e de outras categorias profissionais da área da saúde (Minimel, et al., 2013).

Mesmo diante deste cenário, persiste um distanciamento entre a proposta da PNSTT, o conhecimento teórico e a aplicabilidade das ações de Visat, levando à subnotificação das ocorrências, à fragilidade na vigilância epidemiológica para lidar com absenteísmo, à necessidade de melhorar a notificação de agravos, além de falhas nas ações de Visat do trabalhador de enfermagem e para a prevenção de acidentes (Brasil, 2012; Viana, et al., 2017; Froines, et al., 2011).

Na realidade, evidencia-se que a ferramenta identificada nos estudos brasileiros, o SIMOSTE, nasceu do empenho de pesquisadores, em parceria com hospitais universitários. Porém, ainda que a tecnologia tenha sido cedida para os serviços, parte desses deixou de utilizá-la. Portanto, destaca-se, nesse contexto, a importância de investimentos institucionais na vigilância da saúde dos trabalhadores e necessidade de criação de novos espaços pedagógicos de formação para a vigilância em saúde (Santana, et al., 2013; sias, et al., 2017).

Já na década de 1980 há relatos da proposta de se estabelecer um mínimo de vigilância periódica de saúde do trabalhador, com base em uma avaliação de estilo de vida direcionada algum tipo ferramenta estruturada. A falta de um sistema abrangente de coleta de dados de saúde no trabalho pode resultar em obstáculos para uma intervenção efetiva em saúde ocupacional (Daldon, 2013; Amorim, 2017).

Estudos evidenciam a imprescindibilidade de estratégias de intervenção nas políticas de saúde do trabalhador para oferecer a este profissional uma melhor qualidade de vida, bem como a necessidade de trabalho na cultura de prevenção e ações de vigilância em saúde, voltados para notificação das doenças relacionadas ao trabalho e acidentes de trabalho 
(Ferigollo, et al., 2016; Balista \& Santiago, 2011; Groseclose \& Buckeridge, 2017).

Atualmente, compreende-se que o monitoramento rotineiro compõe um instrumento fundamental para melhorar a prática de saúde coletiva (Baptista, et al., 2011). A coleta de informações contínuas propicia um melhor entendimento da realidade de determinada população trabalhista, suas condições de trabalho e possíveis dificuldades, o que auxilia a oferecer uma assistência de maior qualidade, com um olhar diferenciado e mais atento (Gomez, 2018).

O software Sistema de Monitoramento da Saúde dos Trabalhadores de Enfermagem (SIMOSTE®) foi a única ferramenta eletrônica apontada entre os estudos, tendo sido desenvolvido para captar os agravos à saúde dos trabalhadores de enfermagem e suas consequências. Configura-se como uma inovação tecnológica e seus indicadores dão subsídios para avaliação contínua da saúde desses trabalhadores. Os resultados sugerem que a vigilância/monitoramento é um tema relevante de investigação, porém as ações Vigilância em Saúde do Trabalhador, mesmo que descritas nas legislações vigentes, ainda são insuficientes e, algumas vezes, ineficazes, persistindo a necessidade de formulação de estratégias de formação de agentes capacitados para realizá-las, evitando o distanciamento entre o conhecimento teórico, sua apreensão e aplicação (Baptista, et al, 2011).

\section{Conclusão}

Após análise dos artigos, encontraram-se estudos sobre vigilância do profissional de saúde, entretanto é evidente a escassez de trabalhos sobre monitoramento da saúde do trabalhador de enfermagem.

Evidenciou-se fragilidade na vigilância epidemiológica para lidar com absenteísmo, assim como necessidade de melhoria na área de vigilância em saúde do trabalhador e prevenção de acidentes.

Ao considerar a relevância de melhoria no sistema de vigilância em saúde é necessário desenvolver ações voltadas para o monitoramento das notificações de doenças dos trabalhadores de enfermagem, contribuindo para futuras medidas preventivas e segurança na assistência, como proposto pelo SIMOSTE®.

Sugere-se a necessidade de implementação de ferramentas tecnológicas que favoreçam estratégias de diagnóstico e prevenção, a fim de amenizar e reduzir os agravos à saúde dos trabalhadores de enfermagem.

\section{Referências}

Amorim, L. A., Silva, T. L., Faria, H. P., Machado, J. M. H. \& Dias, E. C. (2017). Worker's surveillance in the primary care: learning with family health team of João Pessoa, Paraíba, Brazil. Ciência e Saúde Coletiva, 22(10), 3403-13. http://doi.org/10.1590/1413-812320172210.17532017

Azaroff, L. S., Levenstein, C. \& Wegman, D. H. (2020). Occupational injury and illness Surveillance: conceptual filters explain underreporting. American Journal of Public Health, 92(9), 1421-9. https://doi.org/10.2105/ajph.92.9.1421.

Balista, S. R.R., Santiago, S. M. \& Filho, H. R. C. (2011). A atenção à saúde do trabalhador nas unidades básicas de saúde do SUS: estudo de caso em Campinas. Revista Brasileira de Saúde Ocupacional, 36(124), 216-26. https://doi.org/10.1590/S0303-76572011000200005.

Baptista, P. C. P., Felli, V. E. A., Mininel, V. A., Karino, M. E., Silva, S. M., Tito, R. S., Peduzzi, M. \& Sarquis, L. M. M. (2011). Using technological innovation as a tool to monitor nursing workers' health. Revista da Escola de Enfermagem da USP, 45, 1621-6. https://doi.org/10.1590/S008062342011000700013

Bernardes, C. L., Vasconcelos, L. H. S., Silva. S, M., Baptista, P. C. P., Felli, V. E. A., Pustiglione, M., Munhoz, R. \& Coa, T. F. (2014). Health problems of nursing workers in a public educational institution. Revista da Escola de Enfermagem da USP, 48(4), 677-83. https://doi.org/10.1590/S0080623420140000400015 .

Brasil. Constituição Federal de 1988. Promulgada em 5 de outubro de 1988. http://www.senado.leg.br/atividade/const/con1988/C ON1988_05.10.1988/ind.asp.

Brasil. Ministério da Saúde. Portaria GM/MS n 3.120, de $1^{\circ}$ de julho de 1998. Instrução Normativa de Vigilância em Saúde do Trabalhador no SUS. Diário Oficial da União 1998.

Brasil. Ministério da Saúde. Portaria No 1.823, de 23 de setembro de 2012. Institui a Política Nacional de Saúde do Trabalhador e da Trabalhadora. http://bvsms.saude.gov.br/bvs/saudelegis/gm/2012/prt1823_23_08_2012.html. 
Brasil. Ministério do Trabalho. Portaria n. ${ }^{\circ}$ 24/GM, de 29 de dezembro de 1994. NR 7, Norma Regulamentadora-7 (1994) Programa de controle médico de saúde ocupacional. Diário Oficial da União, Poder Executivo (1994).

Daldon, M. T. B. \& Lancman, S. (2013). Vigilância em saúde do trabalhador - rumos e incertezas: Revista Brasileira de Saúde Ocupacional, 38(127), 92-106. https://doi.org/10.1590/S0303-76572013000100012

Delgado, L. M. \& Oliveira, B. R.G. (2005). Perfil epidemiológico do adoecimento dos profissionais de enfermagem de um hospital universitário. Nursing São Paulo, 8(87), 365-70. http://bases.bireme.br/cgibin/wxislind.exe/iah/online/?IsisScript=iah/iah.xis\&base=LILACS\&lang=p\&nextAction=lnk\&exp rSearch $=526571 \&$ indexSearch $=$ ID

Donovan, T. L., Moore, K. M. \& VanDenKerhof, E.G. (2008). Employee absenteeism based on occupational health visits in an urban tertiary care canadian hospital. Public health nurse, 25(6), 565-75. http://doi.org/10.1111/j.1525-1446.2008.00744.x.

Ercole, F. F., Melo, L. S. \& Alcoforado, C. L. G. C. (2014) Revisão integrativa versus revisão sistemática. Revista Mineira de Enfermagem, 18(1). http://www.dx.doi.org/10.5935/1415-2762.2014000.

Ferigollo, J. P., Fedosse, E. \& Filha, V. A. V. S. (2016). Qualidade de vida de profissionais da saúde pública. Cadernos Brasileiros de Terapia Ocupacional, 24(3), 497-507. http://dx.doi.org/10.4322/0104-4931.ctoAO0722.

Froines, J. R., Dellenbaugh, C. A. \& Wegman, D. H. (2011). Occupational health surveillance: a mean to identify work-related risks. American Jounal of Public Health, 76(9), 1089-96. http://www.dx.doi.org/10.5935/1415-2762.2014000.

Gomez, C. M., Vasconcellos, L. C. F. \& Machado, J. M. H. (2018). A brief history of worker's health in Brazil's unified health system: progress and challenges. Ciência saúde coletiva, 23(6), 111-22. https://doi.org/10.1590/1413-81232018236.04922018.

Groseclose, S. L. \& Buckeridge, D. L. (2017). Public health surveillance systems: recent advances in their use and evaluation. Annual Review of Public Health, 38(1), 57-79. https://doi.org/10.1146/annurev-publhealth-031816-044348

Guimarães, A. L. O. \& Felli, V. E. A. (2016). Notification of health problems among nursing workers in university hospitals. Revista Brasileira de Enfermagem, 69(3), 507-14. http://dx.doi.org/10.1590/0034-7167.2016690313i.

Mendes, K. D. S., Silveira, R. C. C. P. \& Galvão, C. M. (2008). Revisão integrativa: método de pesquisa para a incorporação de evidências na saúde e na enfermagem. Texto Contexto Enfermagem, 17(4), 758-6. https://doi.org/10.1590/S0104-07072008000400018

Mininel, V. A., Felli, V. E. A., Silva, E. J., Torri, Z., Abreu, A. P. \& Branco, M. T. A. (2013). Workloads, strain processes and sickness absenteeism in nursing. Revista Latino-Americana de Enfermagem, 21(6), 1290-7. http://doi.org/10.1590/0104-1169.2992.2366.

Moher, D., Liberati, A., Tetzlaff, J. \& Altman, D. G. (2009). Preferred reporting items for systematic reviews and meta-analyses: the prisma statement. Plos Medicine 6(7), e1000097. http://doi.org/10.1371/journal.pmed.1000097.

Poole, C. J. M. (1996). A systematic approach to health surveillance in the workplace. Occupational Medicine, 46(3), $245-247$. https://doi.org/10.1093/occmed/46.3.245-a.

Santana, L. L., Sarquis, L. M. M., Miranda, F. M. D., Kalinke, L. P., Felli, V. E. A. \& Mininel, V. A. (2016). Indicadores de saúde dos trabalhadores da área hospitalar. Revista Brasileira de Enfermagem, 69(1), 23-32. http://www.scielo.br/pdf/reben/v69n1/0034-7167-reben-69-01-0030.pdf.

Santana, L. L., Miranda, F. M. D., Karino, M. E., Baptista, P. C. P., Felli, V. E. A. \& Sarquis, L. M. M. (2013). Description of workloads and fatigue experienced among health workers in a teaching hospital. Revista Gaúcha Enfermagem, 34 (1), 64-70. https://doi.org/10.1590/S1983-14472013000100008.

Santana, L. L., Sarquis, L. M. M., Miranda, F. M. A., Kalinke, L. P., Felli, V. E. A. \& Miniel, V. A. (2016). Health indicators of workers of the hospital área. Revista Brasileira de Enfermagem, 69(1), 23-32. http://dx.doi.org/10.1590/0034-7167.2016690104i.

Silva, N. R. (2011). Fatores determinantes da carga de trabalho em uma unidade básica de saúde. Ciência Saúde Coletiva 16(8) $3393-402$. https://doi.org/10.1590/S1413-8123201100090000615.

Sias, N., Cellura, E., Mortara, V., Giacinti, F., Rulfi, A.\& Soru, G. E., et al. (2017). Occupational diseases: when union is not strength. Giornale italiano di medicina del lavoro ed ergonomia, 39(1), 16-25. https://europepmc.org/article/med/29916616.

Trindade, L.R., Merlo, A. R. C., Silva, R. M., Beck, C. L. C. \& Goes, N. C. (2017). Fatores de adoecimento dos trabalhadores da saúde: revisão Integrativa. Ciência e Cuidado da Saúde, 16(4), e39161. http://doi.org/10.4025/cienccuidsaude.v16i4.39161.

Holland, B. J. V., Reneman, M. F., Soer, R., Brouwer, S. \& Boer, M. R. (2018). Effectiveness, and cost-benefit evaluation of a comprehensive workers' health surveillance program for sustainable employability of meat processing workers. Journal of Occupational Rehabilitation, 28(1), 107-20. http://doi.org/10.1007/s10926-017-9699-9.

Vianna, L. C. R., Ferreira, A. P., Vasconcellos, L. C. F. \& Oliveira, M. H. B. (2017). Vigilância em saúde do trabalhador: um estudo à luz da Portaria no 3.120/98. Saúde debate, 41(114), 786-800. https://doi.org/10.1590/0103-1104201711409. 